\title{
DISTÚRBIOS ONDULATÓRIOS DE LESTE (DOLS) NA REGIÃO DO CENTRO DE LANÇAMENTO DE ALCÂNTARA-MA
}

\author{
ELIANE DE CASTRO COUTINHO ${ }^{1}$ e GILBERTO FISCH $^{2 *}$ \\ ${ }^{1}$ Universidade Estadual do Pará (UEPA) \\ Rua Enéas Pinheiro, Belém, PA, CEP 66095-100. \\ E-mail: elianerik@hotmail.com \\ ${ }^{2}$ Centro Técnico Aeroespacial (CTA) \\ * Endereço para correspondência: Praça Marechal Eduardo Gomes, 50, \\ São José dos Campos, SP, CEP 12.228-904. \\ E-mail: gfisch@iae.cta.br
}

Recebido Setembro 2005 - Aceito Fevereiro 2007

\begin{abstract}
RESUMO
Neste artigo foi realizada uma climatologia dos Distúrbios Ondulatórios de Leste (DOLs) que passaram pela região de Alcântara-MA, no período de 1988 a 1997, utilizando-se de um método baseado na mudança do sinal da componente meridional do vento (V). Este método foi aplicado para 3 camadas distintas (entre 850 a $700 \mathrm{hPa}$, entre 700 e $500 \mathrm{hPa}$ e entre 850 a $500 \mathrm{hPa}$ ), objetivando identificar a camada que melhor indicasse a ocorrência dos DOLs. Após a identificação dos eventos de DOLs, estes foram confirmados por um conjunto de dados independentes de cobertura de nuvens (dados ISCCP e imagens infravermelho de satélites GOES-E) e pelos espectros de potência da componente meridional do vento usando as re-análises do ECMWF. Ocorreram eventos de DOLs durante todo o ano com duração de tempo variando de 3 a 6 dias. Esse método mostrou-se adequado para caracterizar os eventos de DOLs e que, durante a época chuvosa, as 3 camadas identificam a passagem do sistema atmosférico. No caso do período seco (jul-dez), as camadas com espessura maior (camadas entre 700 e $500 \mathrm{hPa}$ e entre $850-500 \mathrm{hPa}$ ) conseguiram identificar os eventos, mas não a camada mais baixa (entre 850-500 hPa).
\end{abstract}

Palavras-chave: radiossondagem, vento meridional, ondas de leste.

\begin{abstract}
EASTERLY WAVES DISTURBANCE (DOLs) AT THE REGION OF ALCÂNTARA LAUNCHING CENTER-MA.

A climatology of the easterly waves disturbances (DOLs) over Alcântara - MA was investigated using a method that compute the signal of the observational meridional wind (rawinsoundings from 1988 up to 1997). This technique has been applied to three different layers (850-700, 700-500 and $850-500 \mathrm{hPa}$ ) in order to identify the best layer to indicate the DOLs occurrence. The DOLs events have been confirmed by independent data-sets available: cloud cover (ISPCC data set), infra-red satellite images (from GOES-E) and power spectrum of the meridional component (ECMWF reanalysis). The method detected the presence of the DOLs during the whole year with time-scale of 3-6 days. During the wet period (jan-jun) all three layers identified these atmospheric systems. For the dry season (jul-dec), only the deeper layers (layers between $700-500 \mathrm{hPa}$ and/or 850-500 hPa) were able to identify the events.
\end{abstract}

Keywords: rawinsoundings, meridional wind, easterly waves. 


\section{INTRODUÇÃO}

O clima no Centro de Lançamento de Alcântara (CLA) é muito complexo, pois situa-se na transição entre os climas do Norte do Nordeste Brasileiro (NEB) e da Região Amazônica, envolvendo a influência de vários sistemas atmosféricos diferentes, tais como os Distúrbios Ondulatórios de Leste (DOLs), posição da Zona de Convergência Intertropical (ZCIT), Linhas de Instabilidade (LIs) acoplada com nuvens Cumulonimbus e Vórtices Ciclônicos em Altos Níveis (VCANs). Entre estes sistemas, os DOLs são de extrema importância, por provocarem alterações bruscas das condições sinóticas, principalmente na componente meridional do vento, tendo, como conseqüência, uma umidificação da camada, aumento de nebulosidade e precipitação. Além disso, os sistemas DOLs podem intensificar os sistemas meteorológicos de perturbação convectivas (Barbosa, 2005), tais como as Linhas de Instabilidades que se propagam para o interior da Amazônia ou ainda associar-se com a ocorrência de tempestades tropicais ou mesmo furações na parte norte do Atlântico Equatorial (Cook et al., 2004).

O CLAé o "portal brasileiro para o espaço", pois é o local de onde são lançados os foguetes e veículos espaciais, sendo que o acompanhamento meteorológico durante a fase de montagem, bem como a previsão de tempo para o horário do lançamento, são atividades importantes durante as missões de lançamento de foguetes. É necessário um conhecimento detalhado dos sistemas meteorológicos descritos, visando a melhoria das atividades operacionais de previsão de tempo e que contribuam para o sucesso e segurança dos lançamentos de foguetes.

Em um dos primeiros estudos de meteorologia tropical, Riehl (1945) notou que mudanças na direção do vento acompanhavam a formação e deslocamento de centros isalobáricos e que tais mudanças sugeriam que esses centros eram manifestações da propagação de ondas atmosféricas propagando-se de leste para oeste. A partir de então se definiu este tipo de sistema atmosférico como sendo uma onda de leste. Posteriormente, mudou-se esta denominação para Distúrbios Ondulatórios de Leste.

Este tipo de distúrbio é o mais comum do regime dos ventos alísios, sendo mais freqüente no lado equatorial do cinturão subtropical e mais intenso na média troposfera. Este se desloca pelo Oceano Atlântico até atingir o litoral e a Zona da Mata do NEB (Hastenrath, 1988).

Hall (1989) observou, através da estrutura horizontal de uma onda de leste do Hemisfério Sul - HS (ver Figura 1), que, antes do eixo da onda, a componente meridional do vento (V) era positiva (sul-norte) e que, após a passagem do mesmo, esta se torna negativa (norte-sul). Isso com o eixo inclinado na mesma direção de propagação dos distúrbios, ou seja, no Hemisfério Sul é para oeste e no Hemisfério Norte para leste, devendo-se isto ao sistema de ventos nesta região. Além disso, mostra-se, através da Figura 2, que a maior atividade convectiva encontrava-se exatamente sobre o eixo da onda. Esta mudança na componente do vento é o princípio básico deste trabalho.

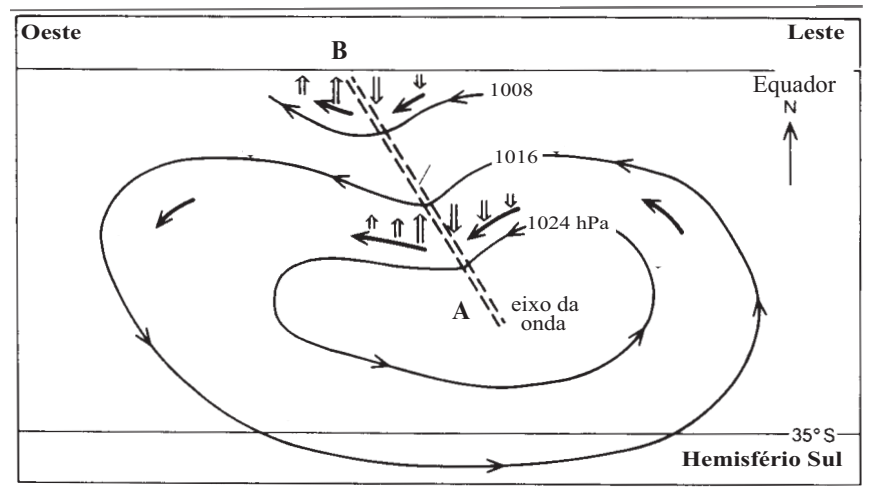

Figura 1 - Estrutura horizontal, em baixos níveis, de uma onda de leste no HS. Fonte: Hall (1989, p.178).

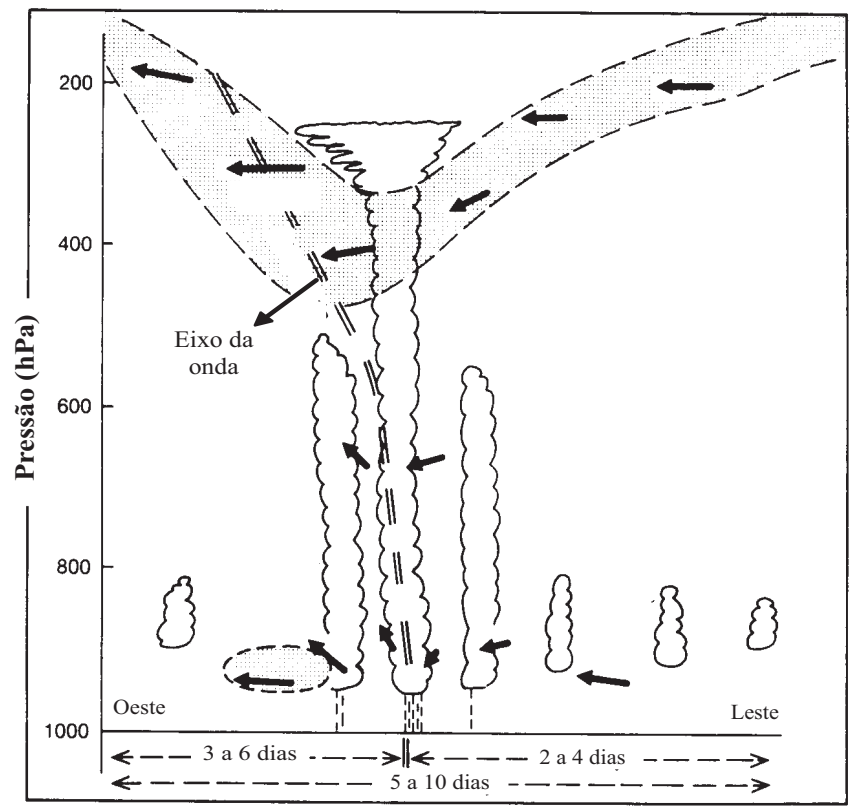

Figura 2 - Estrutura vertical de uma onda de leste no HS. A escala horizontal mostra o tempo aproximado em que a onda utilizou para atravessar a Ilha de Ascenção no Oceano Atlântico. Fonte: Hall (1989, p.178).

Os DOLs possuem 4 regiões distintas, a saber:

- Uma região de crista com tempo bom e visibilidade alta, escoamento divergente na superfície com subsidência e nebulosidade baixa formada por nuvens Cumulus (rasos) de bom tempo;

- Uma região próxima ao eixo do cavado, com nuvens do tipo Cumulus em desenvolvimento, nuvens Cirrus e Altocumulus, visibilidade contínua, porém já com a formação de precipitação esparsa; 
- Uma região no eixo do cavado: presença de nuvens Cumulus congestus, Cirrus e Altocumulus, chuvas frequentes;

- Uma região a leste do cavado, de tempo ruim, com escoamento convergente e fortes movimento ascendentes do ar com a ocorrência de Cumulonimbus e precipitação de moderada para forte.

A Tabela 1, extraída de Coutinho (1999) apresenta vários estudos anteriores referentes aos DOLs, dando ênfase ao número de episódios documentados, bem como a duração de dias e também o nível onde este fenômeno melhor se apresentou. Entretanto, nenhum deles preocupou-se em determinar um indicador da passagem destes sistemas, que pudesse ser utilizado de forma operacional e para previsão de tempo local.

O objetivo deste trabalho é o de determinar uma climatologia da ocorrência de DOLs na região do CLA, através da mudança do sinal da componente meridional do vento observado pelas radiossondagens. Basicamente pretende-se investigar se a técnica da mudança da componente do vento é válida, apresentando-se como um indicador destes eventos, bem como identificar a melhor camada para este método ser utilizado. A aplicação desta metodologia visa melhorar a capacidade operacional de previsão de tempo do Setor de Meteorologia e garantir um aumento de segurança e de sucesso nos lançamentos espaciais do CLA.

\section{DADOS E METODOLOGIA}

\subsection{Dados}

Neste estudo foram utilizados os seguintes conjuntos de dados:

a) Dados das componentes do vento $\left(\mathrm{ms}^{-1}\right)$ e de umidade específica $\left(\mathrm{gkg}^{-1}\right)$ obtidos das radiossondagens do CLA, realizadas às $12: 00 \mathrm{Z}$, durante o período de fevereiro de 1988 a setembro de 1997 (Tabela 2). As informações de vento e de umidade específica foram calculadas a partir das radiossondagens, como sendo o valor aritmético médio para camadas a ser considerada, ou seja, entre 850 e $700 \mathrm{hPa}$ (camada 850-700 hPa), entre 700 e $500 \mathrm{hPa}$ (camada 700-

Tabela 1 - Características dos Distúrbios Ondulatórios de Leste na Região Tropical.

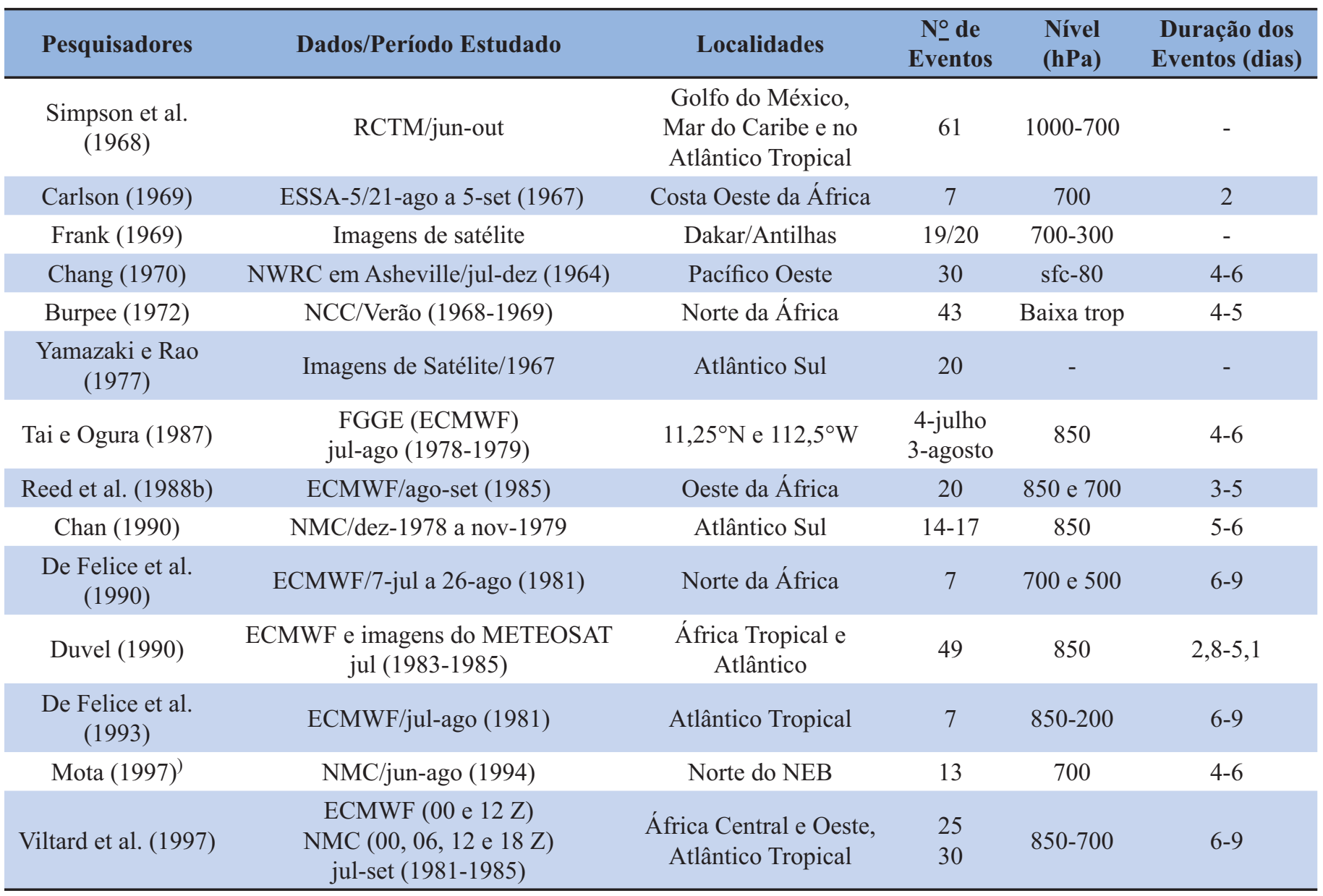


$500 \mathrm{hPa}$ ) e entre 850 a $500 \mathrm{hPa}$ (camada $850-500 \mathrm{hPa}$ ). Estas três camadas foram utilizadas por não existir uma camada ou nível específico em que os DOLs se manifestem mais claramente. Segundo Laurent et al. (1989) as ondas africanas (outra denominação dada às ondas de leste, porém no Hemisfério Norte) no nível de $850 \mathrm{hPa}$ foram mais significativas em um estudo observacional realizado para o ano de 1985, sendo que, para o ano de 1974, os resultados mais significativos foram observados no nível de $700 \mathrm{hPa}$. Normalmente utiliza-se o nível de $500 \mathrm{hPa}$ como indicador de um evento de onda africana (Laurent et al., 1989). Neste trabalho, ao invés de utilizar-se um determinado nível para analisar a ocorrência dos DOLs, buscou-se calcular o valor médio das 3 camadas, pelas observações de radiossondagens.

b) Dados da componente meridional - $\mathrm{V}\left(\mathrm{ms}^{-1}\right)$ das re-análises do ECMWF, às 12:00 Z, entre 1988 a 1993, nos níveis de 850,700 e $500 \mathrm{hPa}$ para o ponto de grade mais próximo do CLA;

c) Dados diários de precipitação (mm), da cidade de São Luiz do Maranhão, durante o período de 1988 e 1997, coletados no Aeroporto de São Luiz pelo DECEA ( Comando da Aeronaútica, Ministério da Defesa);

d) Imagens de infravermelho de satélite GOES-E, entre $1988 \mathrm{e}$ 1997, às 12:00 Z, cedidas pela Divisão de Satélites Ambientais (DSA do CPTEC/INPE de Cachoeira Paulista); e

e) Dados de cobertura total de nuvens (\%) do International Satelitte Cloud Climatology Project (ISCCP), a cada 3 horas, no período de 1988 e 1989.
As informações básicas para este trabalho foram obtidas pelo item $a$, sendo que o restante dos dados foi utilizado para se comprovar a ocorrência (ou não) dos eventos DOLs identificados pelas radiossondagens.

\subsection{Metodologia}

Conforme mostra a Figura 1, a passagem de um DOL provoca uma mudança de direção do vento, principalmente relacionada com a componente meridional do vento. No caso específico do modelo conceitual proposto por Hall (1989), a componente meridional era de Sul (positivo), passando para Norte (negativo), após a passagem do DOL. Além disso, o sistema atmosférico também realiza um transporte atmosférico de vapor d'água. Assim sendo, foram plotados, para todo o período estudado, a variação do sinal da componente $\mathrm{V}$ do vento, associando com a variação da umidade específica, nas três camadas citadas, visando identificar padrões do comportamento atmosférico que atuam na região do CLA. Este método busca apresentar um indicador da passagem de DOLs sobre este região e também foi utilizado por Martins et al. (2002) para a identificação de DOLs na costa Atlântica do estado do AMAPÁ, durante o mês de agosto de 2001. A Figura 3 apresenta um exemplo para o ano de 1988.

Após a identificação dos DOLs, verificou-se a existência de aglomerados de nuvens nas imagens infra-vermelho de satélite do GOES-E e também um aumento de valores máximos de nebulosidade nos dados do ISCCP. Este procedimento foi realizado para comprovar a existência ou não dos DOLs em

Tabela 2 - Freqüência das Radiossondagens Diárias no CLA.

\begin{tabular}{cccccccccccc}
\hline & $\mathbf{1 9 8 8}$ & $\mathbf{1 9 8 9}$ & $\mathbf{1 9 9 0}$ & $\mathbf{1 9 9 1}$ & $\mathbf{1 9 9 2}$ & $\mathbf{1 9 9 3}$ & $\mathbf{1 9 9 4}$ & $\mathbf{1 9 9 5}$ & $\mathbf{1 9 9 6}$ & $\mathbf{1 9 9 7}$ & Total \\
\hline Jan & - & - & 09 & - & 19 & - & 26 & 26 & 25 & 26 & 131 \\
\hline Fev & 05 & - & - & - & 25 & - & 26 & 11 & 27 & 22 & 116 \\
\hline Mar & 24 & - & - & - & 27 & 06 & 30 & 15 & 23 & 21 & 146 \\
\hline Abr & 23 & - & - & - & 28 & 24 & 26 & 15 & 24 & 28 & 168 \\
\hline Mai & 26 & - & - & - & 24 & 27 & 31 & 15 & 11 & 29 & 163 \\
\hline Jun & 24 & 15 & - & - & 18 & 28 & 27 & 07 & 15 & 29 & 163 \\
\hline Jul & 28 & 17 & - & - & 29 & 20 & 28 & 25 & 25 & 31 & 203 \\
\hline Ago & 24 & 25 & - & - & 29 & 10 & - & 17 & 18 & 29 & 152 \\
\hline Set & 23 & 08 & 14 & - & 25 & 30 & - & 29 & 23 & 29 & 181 \\
\hline Out & 29 & 21 & 11 & 16 & 25 & 28 & - & 25 & 23 & - & 178 \\
\hline Nov & 28 & 23 & - & 26 & 28 & 23 & 25 & 03 & 20 & - & 176 \\
Dez & 11 & 29 & - & 10 & 09 & 27 & 27 & 25 & 28 & - & 166 \\
\hline Total & 245 & 138 & 34 & 52 & 286 & 223 & 246 & 213 & 262 & 244 & 1943 \\
\hline
\end{tabular}



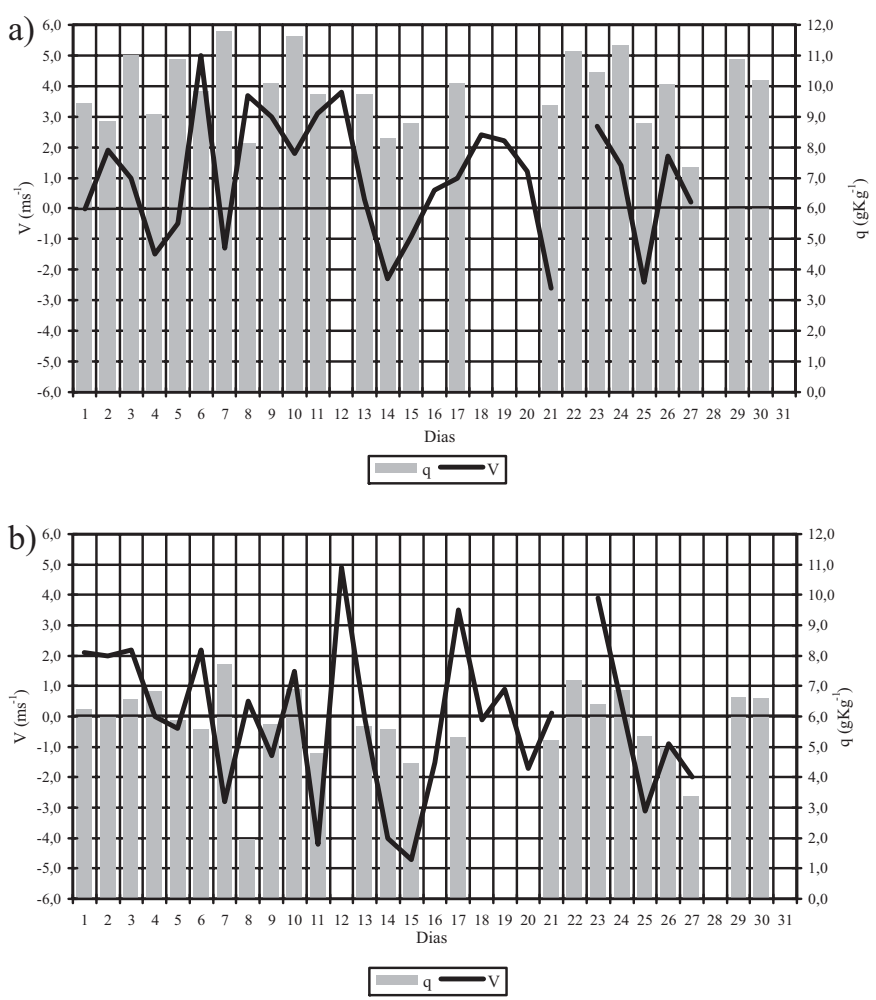

Figura 3 - Relação de V com o aumento da umidade específica no CLA, no mês março de 1988. (a) em $850-700 \mathrm{hPa}$; e (b) em 700-500 hPa.

dois outros conjuntos de dados independentes (no caso, imagens de satélites GOES e dados do ISCCP). Utilizou-se também a análise espectral da componente meridional das re-análises do ECMWF, pois estes são contínuos no tempo, visando assim obter o espectro de potência do vento e associá-lo com os eventos de DOLs detectados previamente sobre a região do CLA.

Os espectros de energia da componente meridional do vento foram determinados pelo algoritmo da Fast Fourier Transforms (FFT). Nesta transformada foi aplicado o janelamento de "Hamming", para diminuir a variância das séries (Marple Jr, 1987).

\section{RESULTADOS E DISCUSSÃO}

A climatologia da ocorrência de DOLs foi realizada da seguinte forma: inicialmente, com os valores originais extraídos das radiossondagens, nas camadas de $850-700 \mathrm{hPa}, 700-500 \mathrm{hPa}$ e 850-500 hPa, calculou-se o valor médio da componente meridional do vento para cada dia, obtendo a série temporal desta variável e associando-a com o aumento ou diminuição da umidade específica (q) relativa ao valor médio do período. Foram considerados como eventos de DOLs aqueles em que ocorreram mudanças de sinal de $\mathrm{V}$ associadas com anomalias positivas de $q$, em períodos persistentes de 3-10 dias. Esse intervalo foi determinado como período típico dos DOLs no NEB por outros autores, tais como Espinosa (1996) e Mota (1997).

As Tabelas 2, 3 e 4 apresentam a distribuição dos DOLs entre $850-700 \mathrm{hPa}, 700-500 \mathrm{hPa}$ e $850-500 \mathrm{hPa}$, respectivamente, apresentando 100 eventos na camada de 850 $500 \mathrm{hPa}, 96$ casos na de 700-500 hPa e 64 casos na camada de $850-700 \mathrm{hPa}$, considerando-se todo o período de dados de radiossondagem disponível (1988-1997). Todos os eventos classificados pela mudança da direção do vento foram checados individualmente. A seguir, descreve-se as características principais da variabilidade da ocorrência dos DOLs para as 3 camadas consideradas.

\subsection{Camada de 850-700 hPa}

O total de eventos de DOLs na camada de 850-700 hPa foi de 64 casos. Os anos de 1990 e 1991 possuem, cada um, somente 3 meses de radiossondagens (Tabela 3 ), sendo que em 1990, detectou-se a passagem de apenas 1 DOL (setembro). A Tabela 3 também mostra que o ano de 1993, apesar de ter uma quantidade maior de radiossondagens com 10 meses de dados, a ocorrência de DOLs foi pequena, com somente 2 eventos. Já os anos de 1988 e 1994 obtiveram os máximos valores, com 10 e 17 eventos, respectivamente. Classificou-se também os dados pelo número de ocorrência por trimestre e pelas estações chuvosa (jan-jun) e seca (jul-dez), segundo a climatologia do local.

Verificou-se ainda que o trimestre de ago-set-out é o que obtém a menor quantidade de DOLs, com 1 evento em cada mês. Já o máximo ocorre nos meses de março, abril e dezembro com totais de 8, 10 e 8 eventos, respectivamente. Além disso, nota-se que a estação chuvosa apresenta a maior concentração da passagem desses distúrbios, com total de 42 eventos, restando 22 eventos de DOLs na estação seca.

O período de 1994 a 1997 foi bastante ativo, com um total de 37 eventos de DOLs. Já entre os anos de 1988 e 1993 a quantidade de DOLs apresenta uma variabilidade alta, alternando-se anos com muitos casos (1988) e anos com pouco ou nenhum eventos (1990, 1991 e 1993). As imagens de satélite, juntamente com os dados do ISCCP, confirmam a existência de aglomerados de nuvens nos dias em que os eventos de DOLs sobre Alcântara foram detectados. A nebulosidade é alta com valores entre 80 e $100 \%$ de cobertura de nuvens, conforme mostra a Figura 4 para o mês de março de 1988.

\subsection{Camada de 700-500 hPa}

O número total de DOLs encontrado na camada de 700-500 hPa foi de 96 eventos, apresentando uma forte variabilidade interanual (Tabela 4). Já a distribuição sazonal é mais uniforme. 
Tabela 3 - Distribuição dos DOLs entre 850-700 hPa

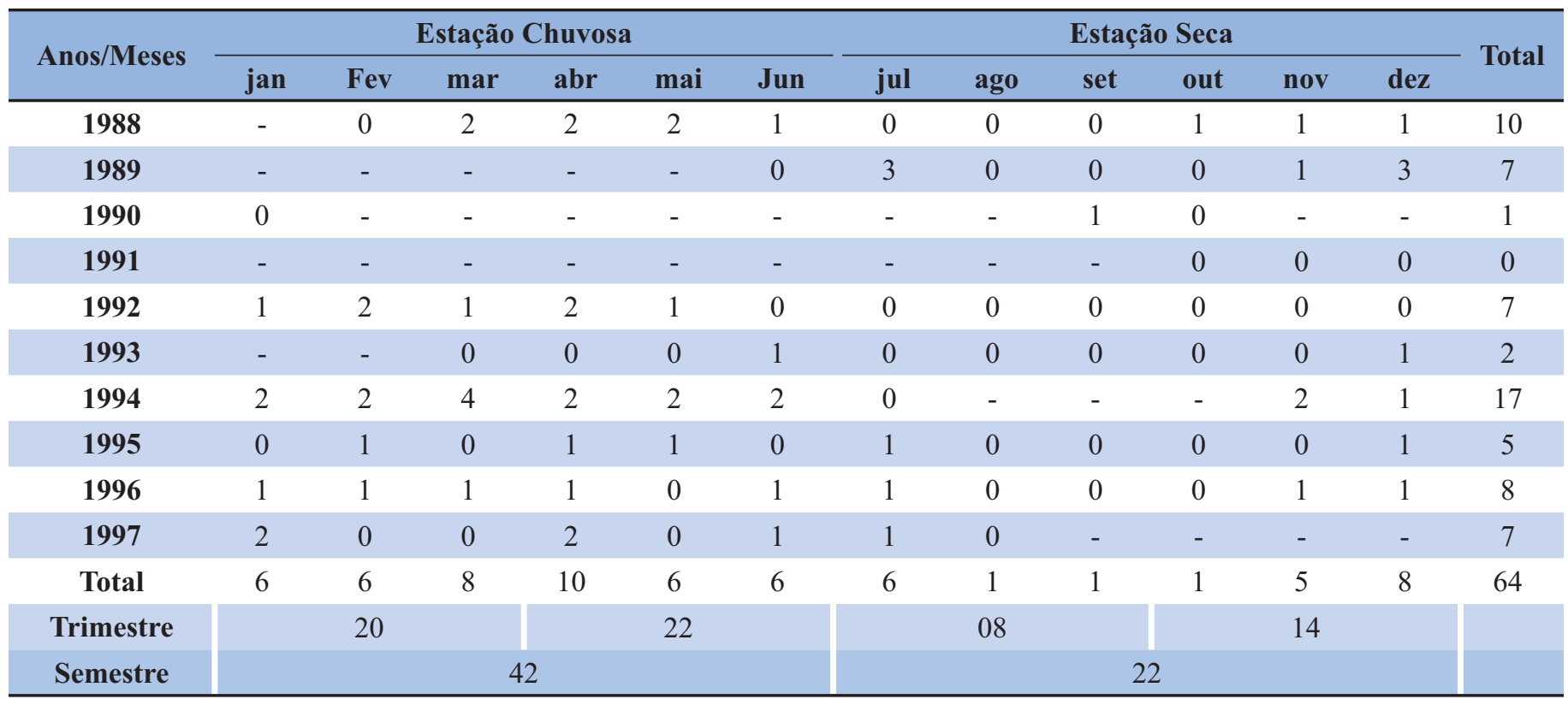

“-”: representa meses com falta dos dados de radiossondagens.

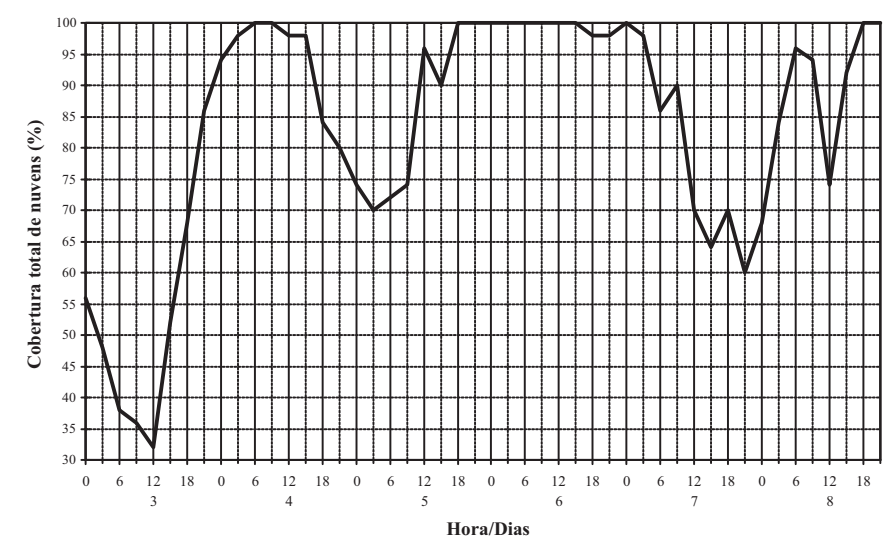

Figura 4 - Cobertura total de nuvens entre os dias 3-8 de março de 1988, em 8 horários.

Como já foi mencionado na camada de $850-700 \mathrm{hPa}$, os anos de 1990 e 1991 apresentam somente 3 meses com radiossondagens, com 4 eventos de DOLs, 3 em 1990 (Jan-Set-Out) e 1 caso em 1991 (outubro). A Tabela 4 também mostra que os anos com maior ocorrência desses distúrbios foram os de 1988 (19 casos) e de 1994 (21 anos).

A distribuição mensal foi realizada para determinar a sazonalidade dos eventos de DOLs, verificando que o trimestre de jul-ago-set é o que apresenta a maior ocorrência desses eventos, com total de 27 casos, sendo que o trimestre abr-mai-jun tem a menor ocorrência com 20 eventos (Tabela 4). Essa tabela ainda mostra que a estação seca possui uma concentração de DOLs sobre Alcântara levemente superior a da estação chuvosa, com 51 eventos. Dos 27 eventos ocorridos em 1988 e 1989, somente 4 casos apresentaram cobertura de nuvens, em algum horário, abaixo de $20 \%$, sendo que, 15 desses eventos possuem cobertura total acima de $90 \%$.

\subsection{Camada de $850-500 \mathrm{hPa}$}

Esta camada concentra o maior número de casos de DOLs, ao ser comparada com as camadas anteriormente descritas, com um total de 100 eventos. Os anos de 1990 e 1991 possuem as mesmas características apresentadas em 850-700 hPa. A Tabela 5 também mostra que 1988 e 1994 apresentam as quantidades máximas de DOLs, com 22 e 16 eventos, respectivamente e entre 1992 e 1997 a quantidade de DOLs foi a mesma.

\subsection{Comparação entre as Camadas de 850-700 hPa, 700-500 hPa e 850-500 hPa e Variabilidade Interanual}

A presença de DOLs pode ocorrer em vários níveis para diferentes anos (Laurent et al., 1989). Assim sendo utilizouse as camadas de $850-700 \mathrm{hPa}, 700-500 \mathrm{hPa}$ e $850-500 \mathrm{hPa}$ como potenciais indicadores dos DOLs. Essas três camadas apresentam muitas diferenças e similaridades para estudar a ocorrência dos DOLs sobre Alcântara, mostrando variabilidade tanto na distribuição sazonal como na interanual. Além disso, elas também apresentam espessuras diferentes, associados com a profundidade do sistema e da convecção. 
Tabela 4 - Distribuição dos DOLs entre 700-500 hPa.

\begin{tabular}{|c|c|c|c|c|c|c|c|c|c|c|c|c|c|}
\hline \multirow{2}{*}{ Anos/Meses } & \multicolumn{6}{|c|}{ Estação Chuvosa } & \multicolumn{6}{|c|}{ Estação Seca } & \multirow{2}{*}{ Tota } \\
\hline & jan & Fev & mar & abr & mai & Jun & jul & ago & set & out & nov & dez & \\
\hline 1988 & - & 0 & 2 & 2 & 1 & 1 & 3 & 2 & 2 & 3 & 2 & 1 & 19 \\
\hline 1990 & 1 & - & - & - & - & - & - & - & 1 & 1 & - & - & 3 \\
\hline 1991 & - & - & - & - & - & - & - & - & - & 1 & 0 & 0 & 1 \\
\hline 1993 & - & - & 0 & 0 & 1 & 1 & 1 & 0 & 1 & 1 & 1 & 1 & 7 \\
\hline 1994 & 4 & 2 & 3 & 2 & 4 & 1 & 1 & 1 & - & - & 3 & 0 & 21 \\
\hline 1995 & 0 & 0 & 2 & 1 & 1 & 1 & 1 & 0 & 1 & 0 & 0 & 0 & 7 \\
\hline 1996 & 2 & 2 & 2 & 0 & 1 & 2 & 2 & 0 & 2 & 1 & 2 & 0 & 16 \\
\hline Semestre & \multicolumn{6}{|c|}{45} & \multicolumn{6}{|c|}{51} & \\
\hline
\end{tabular}

Tabela 5 - Distribuição dos DOLs entre 850-500 hPa.

\begin{tabular}{|c|c|c|c|c|c|c|c|c|c|c|c|c|c|}
\hline \multirow{2}{*}{ Anos/Meses } & \multicolumn{6}{|c|}{ Estação Chuvosa } & \multicolumn{6}{|c|}{ Estação Seca } & \multirow{2}{*}{ Total } \\
\hline & jan & Fev & mar & abr & mai & Jun & jul & ago & set & out & nov & dez & \\
\hline 1988 & - & 0 & 3 & 2 & 2 & 1 & 2 & 3 & 2 & 4 & 2 & 1 & 22 \\
\hline 1990 & 0 & - & - & - & - & - & - & - & 1 & 0 & - & - & 1 \\
\hline 1991 & - & - & - & - & - & - & - & - & - & 0 & 0 & 0 & 0 \\
\hline 1993 & - & - & 0 & 0 & 2 & 1 & 1 & 0 & 3 & 1 & 0 & 1 & 9 \\
\hline 1994 & 1 & 2 & 1 & 2 & 2 & 3 & 2 & 1 & - & - & 2 & 0 & 16 \\
\hline 1995 & 1 & 0 & 1 & 1 & 0 & 0 & 3 & 1 & 2 & 1 & 0 & 2 & 12 \\
\hline 1996 & 3 & 2 & 1 & 1 & 0 & 2 & 3 & 0 & 1 & 0 & 2 & 0 & 15 \\
\hline Semestre & \multicolumn{6}{|c|}{44} & \multicolumn{6}{|c|}{56} & \\
\hline
\end{tabular}

As Tabelas 3, 4 e 5 mostram que a máxima ocorrência de eventos de DOLs concentram-se nos anos de 1988 e de 1994, para todas as camadas. Contudo, a camada de 850$500 \mathrm{hPa}$ apresenta o seu maior valor para o ano de 1988 e as outras duas camadas no ano de 1994. A maior similaridade da ocorrência desses distúrbios é entre as camadas de 700-500 hPa e 850-500 hPa. Já a menor ocorrência desses eventos aconteceu nos anos de 1990 e 1991, coincidentes nas três camadas. Ressalta-se de que o número de radiossondagens, ao longo dos anos, não foi igual: os anos de 1990 e 1991 tiveram apenas 34 e 42 sondagens, respectivamente. Entretanto, os anos de 1988 e 1994 tiveram aproximadamente 245 sondagens, enquanto que nos anos de 1992, 1996 e 1997 foram realizadas 286, 262 e 244 sondagens, respectivamente. Estas análises mostram que os DOLs ocorrem durante o ano todo, conforme os resultados também encontrados por Chan (1990), para 1 ano de análises do NMC (atual NCEP) e Espinosa (1996), para 10 anos de dados do ECMWF. 
Além disto, essas tabelas mostram que, apesar da camada de $850-700 \mathrm{hPa}$ apresentar a menor quantidade total de DOLs (64 eventos), a ocorrência desses, na estação chuvosa (42 eventos), é quase que a mesma encontrada nas camadas de 700-500 (45 eventos) e $850-500 \mathrm{hPa}$ (44 eventos). Sendo assim, pode-se afirmar que não existem diferenças significativas nas quantidades de distúrbios entre essas três camadas, para os meses entre janeiro e junho, que representam a estação chuvosa.

Já a estação seca mostra diferenças marcantes entre as três camadas, pois a de 700-500 (51 eventos) e de 850-500 hPa (56 eventos) apresentam mais do que o dobro de DOLs encontrados em $850-700 \mathrm{hPa}$ (22 eventos). Com isso pode-se fazer a hipótese de que esses distúrbios, na estação seca, ocorrem em altitudes maiores e são melhores detectados nas camadas de 700-500 hPa e 850-500 hPa.

O período de duração dos DOLs é semelhante entre as três camadas estudadas, apresentando valor médio de 4,1 dias para a camada entre $850-700 \mathrm{hPa}, 4,6$ dias para a camada entre 700-500 hPa e 4,7 dias na camada de 850-500 hPa, coincidindo com outros autores, tais como: Kagano (1979), Nita (1985) e Espinosa (1996).

\subsection{Ocorrência de DOLs e Anomalias de Precipitação}

A passagem dos DOLs influenciam a precipitação da região, conforme descrito nas Figuras 1 e 2. As Figuras 5 representam a relação de anomalias dos totais anuais de precipitação com a passagem dos DOLs sobre o CLA. Esta figura mostra que os anos (1990, 1991, 1992 e 1993), com baixa freqüência de DOLs ( 0 -2 eventos por mês), apresentaram anomalias anuais negativas de precipitação. Por outro lado, os anos de 1988, 1989 e 1994, nos quais detectou-se uma quantidade de DOLs entre 2-4 eventos mensal, mostram que a precipitação na região foi maior, principalmente nos anos de 1989 e 1994 (mais ativos). Isto é um indício de que esses distúrbios podem estar associados a episódios de El Niño (EN) e La Niña (LN), ou seja, maior freqüência de DOLs em anos de LN e menor freqüência em anos de EN (Figura 5a). Gu e Zhang (2001), citados por Cook et al. (2004), mostraram resultados de que as ondas africanas (DOLs no hemisfério norte) não se correlacionam com eventos ENSO. Porém, Grist (2002), também citado por Cook et al. (2004), mostrou que estes sistemas provocam muita variabilidade interanual, exemplificando os anos de 1994 como um ano pouco ativo de ondas (e também seco) e o ano de 1995 como sendo muito ativo, com altas precipitações. A página www.nhc.noaa.gov (do Centro Nacional de Furacões dos EUA) apresenta uma estatística do número de distúrbios tropical por ano, sendo que ocorreram 7 distúrbios tropicais no Atlântico (tempestades e furacões) para o ano de 1994 e 19 casos em 1995. Se observar a ocorrência de
DOLs no NEB para estes anos, nota-se de que o ano de 1994 teve a ocorrência de muitos DOLs (sempre superior ao número de 16 eventos para as 3 camadas), ao passo que o ano de 1995 ocorreram poucos casos: menos que 7 casos para as camadas mais baixas. Será que existe um mecanismo na atmosfera de propagação de energia na forma de distúrbios tropicais para o hemisfério norte ou através de ondas de leste para o hemisfério sul? Isto somente poderá ser investigado através de dados de re-análises, pela continuidade temporal das informações e não foi objeto de estudo nesta pesquisa.

No final da década de 80 e no início dos anos 90, ocorreram dois episódios do evento LN (anos de 1988-1989 e 1995-1996) e um episódio do evento EN (1990-1994) (Stormax, 1998). Alves et al. (1997) verificaram que em anos de LN, além dos altos índices de precipitação do NEB e baixa Temperatura da Superfície do Mar (TSM) no Oceano Pacífico Leste, ocorreram anomalias positivas da Pressão ao Nível Médio do Mar (PNM) na Bacia do Atlântico Norte, que favorecem a intensificação dos alísios de nordeste. Este fato impulsiona a ZCIT para posições próximas à costa norte do NEB, e conseqüentemente favorecendo a ocorrência de chuvas abundantes (Figura 5b). Mas não se sabe se isto está relacionado com um maior número de DOLs ou não.

\subsection{Associação dos DOLs com outros Fenômenos Atmosféricos tais como ZCIT, VCAN e LCbs}

A posição geográfica da ZCIT pode ser identificada pela componente meridional do vento (Ferreira, 1998), influenciando as características climáticas da região de Alcântara durante os meses de janeiro a março. Entretanto esse sistema não interferiu na identificação de eventos dos DOLs, pois estes eventos apresentam duração média menor, em torno de 4,5 dias. Além disto a variação da nebulosidade observada na ZCIT é normalmente associada com ondas equatoriais, localizando-se no HN durante a estação chuvosa do HS. Portanto não afeta, de maneira direta, a precipitação do NEB. Segundo Tai e Ogura (1987) os DOLs aparecem com mais intensidade durante o período de atuação da ZCIT e isto foi comprovado pelos resultados das Tabelas 3,4 e 5 .

No caso dos VCANs, Gan (1982), Kayano et al. (1997) e Calbete et al. (1998), entre outros, afirmam que a maior freqüência ocorre nos meses de verão (dez-jan-fev), e que somente $10 \%$ desses sistemas atingem a superfície. Assim sendo, a possibilidade de que esses sistemas possam se confundir com os DOLs foram checados e constatou-se que não havia a presença de VCANs nos dias da passagem dos DOLs sobre Alcântara.

As LCbs se formam tanto na estação chuvosa como na seca, porém na estação chuvosa essas linhas são mais intensas, localizando-se sobre o norte do NEB. Durante a estação seca 


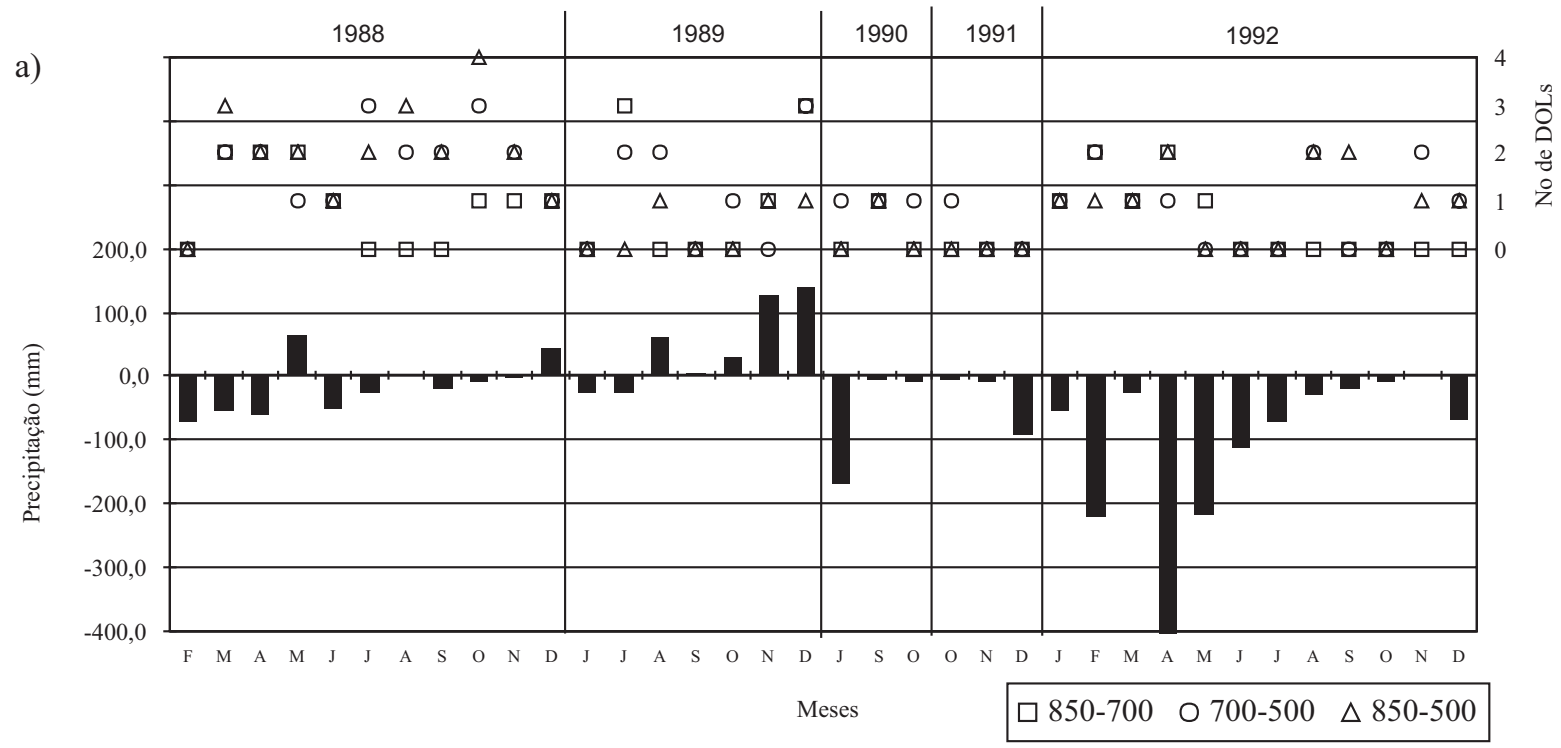

b)

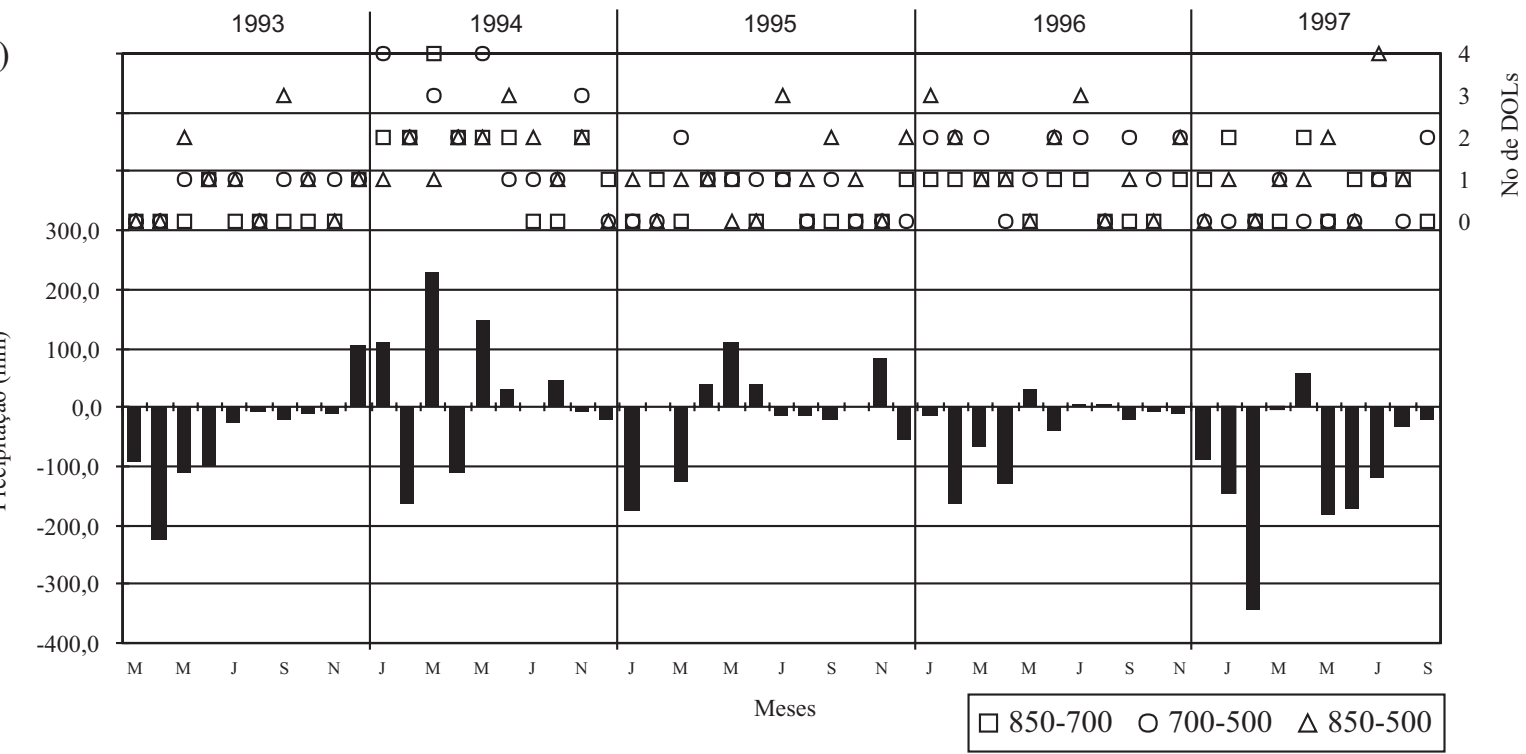

Figura 5 - Associação dos DOLs com anomalias de precipitação (PRP de São Luiz-PRP média). (a) anos de 1988 a 1992; e (b) anos de 1993 a 1997.

as LCbs são mais fracas e permanecem ao norte da América do Sul. Além disto, tem-se a duração das LCbs, que aparecem e desaparecem subitamente (Cohen, 1989) e a dos DOLs, que permanecem sobre Alcântara entre 3-6 dias, podendo chegar a 10 dias.

\section{CONCLUSÕES}

A análise da mudança do sinal de $\mathrm{V}$ detectou a presença de DOLs ao longo do ano inteiro, com intervalo de tempo variando de 3 a 10 dias. Contudo, em $85-90 \%$ do total dos eventos, a maior frequiência ocorreu entre 3-6 dias. Esse método ainda revelou 64 eventos na camada de $850-700 \mathrm{hPa}, 96$ casos em 700-500 hPa e 100 eventos na camada de $850-500 \mathrm{hPa}$, sendo que todos apresentaram anomalias positivas da umidade específica, além de nebulosidade sobre a região de Alcântara.

A maior freqüência dos DOLs entre $850-700 \mathrm{hPa}$ ocorre na estação chuvosa, com quase o dobro da quantidade existente na estação seca. Já entre 700-500 hPa e 850-500 hPa a estação seca é a que apresenta a maior quantidade de DOLs, porém com variação pequena em relação a estação chuvosa. Além disso, os trimestres de jul-ago-set (700-500 hPa), jan-fev-mar e 
out-nov-dez, entre 850-500 hPa são os que concentram a maior quantidade de DOLs.

De modo geral a estação seca mostra diferenças muito marcantes entre as três camadas. Com isso sugere-se a hipótese de que esses distúrbios, na estação seca, são melhores detectados nas camadas de 700-500 e 850-500 hPa.

Nos meses de janeiro a junho (estação chuvosa) não existem diferenças significativas nas quantidades de distúrbios entre essas três camadas. Outra semelhança detectada entre as camadas de 850-700, 700-500 e 850-500 hPa é a variabilidade interanual, sendo que a maior quantidade de distúrbios concentraram-se nos anos de 1988 e 1994.

\section{REFERÊNCIAS BIBLIOGRÁFICAS}

ALVES, J.M.B.; SOUZA, E.B.; REPELLI, C.A.; VITORINO, M.I.; FERREIRA, N.S. Episódios de La Niña na Bacia do Oceano Pacífico Equatorial e a distribuição sazonal e intrasazonal das chuvas no setor norte do nordeste brasileiro. Revista Brasileira de Meteorologia, v.12, n.1, p.63-76, 1997.

BARBOSA, R.L. Interação das perturbações convectivas iniciadas na costa Norte do Brasil com Distúrbios Ondulatórios de Leste. São José dos Campos. 2005. 81 p. Dissertação (Mestrado em Meteorologia) - Instituto Nacional de Pesquisas Espaciais - INPE.

CALBETE, N.O.; GAN, M.A.; SATYAMURTY, P. Vórtices de alta troposfera que atuam sobre a região nordeste do Brasil. [online]. http://www.cptec.inpe.br/products/ climanalise/ cliesp10a/dock.html. Jun. 1998a.

CHAN, C.S. Análise de distúrbios ondulatórios de leste sobre o Oceano Atlântico Equatorial Sul. São José dos Campos. 1990. 134p. Dissertação (Mestrado em Meteorologia) - Instituto Nacional de Pesquisas Espaciais - INPE.

COHEN, J.C.P.; SILVA DIAS, M.A.F.; NOBRE, C.A. Aspectos climatológicos das linhas de instabilidade na Amazônia. Climanálise, v.4, n.11, p.34-40, 1989.

COOK, K.H.; PATRICOLA, C.M.; VIZI, E.K. Easterly Waves in the tropical Atlântic: climatology and variability. Monthly Weather Review (submetido), 2004.

COUTINHO, E.C. Estudo das características atmosféricas na região do Centro de Lançamento de Foguetes de Alcântara (CLA). São José dos Campos. 1999. 141p. Dissertação (Mestrado em Meteorologia) - Instituto Nacional de Pesquisas Espaciais - INPE.
ESPINOSA, E.S. Distúrbios nos ventos de leste no atlântico tropical. São José dos campos. 1996. 127p. Dissertação (Mestrado em Meteorologia)-Instituto Nacional de Pesquisas Espaciais-INPE.

FERREIRA, N. S. Zona de Covergência Intertropical. [online]. http:/www.cptec.inpe.br/products/climanalise/ cliesp10a/acit_1.html>.jun. 1998.

GAN, M.A. Um estudo observacional sobre as baixas frias da alta troposfera, nas latitudes subtropicais do Atlântico Sul e leste do Brasil. São José dos Campos. 65p. (INPE2685-TDL/126). Dissertação (Mestrado em Meteorologia) - Instituto Nacional de Pesquisas Espaciais, 1982.

HALL, B.A. Westward-moving disturbances in the South Atlantic coinciding with heavy rainfall events at Ascension Island. Meteorololgy Magazine, v.118, p.175-181, 1989.

HASTENRATH, S. Climate and circulation of the tropics. New York Atmospheric Sciences Library, 1988, 455p.

KAGANO, M.T. Um estudo climatológico e sinótico utilizando dados de radiossondagem (1968-1976) de Manaus e Belém. São José dos Campos. 111p. (INPE1559-TDL/013). Dissertação (Mestrado em Meteorologia) - Instituto Nacional de Pesquisas Espaciais, 1979.

KAYANO, M.T.; FERREIRA, N.J.; RAMIREZ, M.C.V. Summer circulation patters related to the upper tropospheric vortices over the tropical South Atlantic. Meteorology and Atmospheric Physics. v. 64, n. 3-4, p. 203-213, 1997.

LAURENT, H.; VILTARD, A.; De Felice, P. Performance evalution and local adaptation of the ECMWF system forecasts over northern Africa for summer 1985. Monthly Weather Review, v.117, p.1999-2009, 1989.

MARPLE JR., Jr., S.L. Digital Spectrum Analysis with Applications. Prentice Hall Signal Processing Series, 87, 492 p.

MARTINS, A.C. da S.; ROCHA, E.J.P.; LIMA, K.C. Análise da componente meridional do vento durante a ocorrência de um Distúrbio Ondulatório de Leste na costa Atlântica do Estado do Pará. XII Congresso Brasileiro de Meteorologia, Foz de Iguaçu, PR, agosto de 2002. CD-ROM, p. 3721 -3728, 2002. 
MOTA, G.V. Estudo observacional de distúrbios ondulatórios de leste no nordeste brasileiro. São Paulo. 1997. 92p. Dissertação (Mestrado em Meteorologia) - Instituto Astronômico e Geofísico - USP.

NITA, T.; NAGAKOMI, Y.; SUZUKY, Y.; HASEGAWA, N.; KAOKURA, A. Global Analysis of the lower tropospheric disturbances in the tropics during the northern summer of the FGGE year. Part I: global features of the disturbances. Journal of the Meteorological Society of Japan. v.63, n.1, p.1-19, 1985.
RIEHL, H. Waves in the easterlies and polar front in the tropics. Chicago: Chicago University, Departament of Meteorology, 1945. 79p.

STORMAX. EI Niño and La Niña. [online]. <http://www. stormax.com/ lanina.htm>. Abr. 1999.

TAI, K.S.; OGURA, Y. An observational study of easterly waves over the eastern Pacific in the northern summer using FGGE data. Journal Atmospheric Sciences, v.44, n.2, p.339-361, 1987. 